\title{
Drainage of Masseteric Abscess via Sublabial Approach: A Case Series Xing Yi $Y^{1}$, Sazly $J^{2}$, Zakaria $Z^{3}$, Choo $P K^{4}$
}

\begin{abstract}
:
Masseteric abscesses are usually drained through the external or trans-oral approach. Both choices have their own risks and benefits. We included in our case series 3 patients whom presented with painful facial swelling and were diagnosed as masseteric abscess. We performed the sublabial approach to drain the collection. Post-operatively, they recovered well and there were no recurrences. This technique and outcome of surgery are described.
\end{abstract}

Key words: masseteric abscess; sublabial approach; incision and drainage

Bangladesh Journal of Medical Science Vol. 15 No. 03 July’16. Page : 492-494

\section{Introduction:}

Masseteric abscess is defined as pus collection in the masseter space, and it is an uncommon type of deep neck abscess. It is most commonly from dental origin especially from the lower molar, followed by infection from needle injection to the inferior alveolar nerve when the needle is misdirected to the lateral ramus resulting in infection from an osteomyelitic zygomatic or temporal bone. ${ }^{1}$ Treatments of this disease are by administering appropriate antibiotics and surgical drainage via trans-oral or external approach although needle aspiration can be the initial method. ${ }^{2}$ We present a case series in which our patients underwent sublabial incision and drainage, sharing our experience in the outcome of surgery via this approach.

Case 1: A 5 year old Indian girl, who presented to us with right infraorbital swelling secondary to sinusitis, post operative day eleven endoscopic sinus decompression (right maxillary antrostomy, uncinectomy, anterior ethmoid antrostomy), complained of an increasing right facial swelling for three days, associated with pain and fever. On examination, there was a superficial swelling at the right cheek with overlying skin inflammation. There was no trismus nor was there buccal swelling intraorally. Patient subsequently underwent a sublabial approach incision and drainage and 8 milliliters of pus was drained from the buccal space. Patient underwent re-incision and drainage two days later due to persistent swelling via a similar approach, but only drained 10 milliliters hemoserous fluid. Patient was started on intravenous clindamycin and discharged well with oral clindamycin. Patient was seen two weeks later during follow up clinic and was well.

Case 2: A 13 year old Indian boy, presented with one month history of painful, progressively enlarging left facial swelling, preceded by left lower alveolar toothache few days prior, associated with fever. Clinical examination revealed a warm and tender swelling at the left cheek measuring $7 \times 7 \mathrm{~cm}$. There was trismus, but intraoral examination did not demonstrate medialization. Flexible nasopharyngolaryngoscopy was clear. Aspiration was done and 3 milliliters of pus was drained. Patient underwent incision and drainage via a sublabial approach, draining 5 milliliters of pus medial to left masseter muscle. Patient was discharged well after five days in ward. Patient was reviewed two weeks later and was well.

Case 3: A patient with a history post dental extraction presented with an increasing painful right facial swelling for one week. Clinical examination revealed trismus less than one finger breath and a 4 by 4 centimeter swelling at the angle of right mandible.

1. Yeoh Xing Yi*,

2. Jamal Sazly,

3. Zahirrudin Zakaria,

4. Pua Kin Choo.

Department of Otorhinolaryngology, Penang General Hospital, Georgetown, Pulau Pinang, Malaysia.

Corresponds to: Dr Yeoh Xing Yi, Department of Otorhinolaryngology, Penang General Hospital , Jalan Residensi , 10990 Georgetown, Pulau Pinang, Malaysia.

Email: xy.yeoh@gmail.com. 


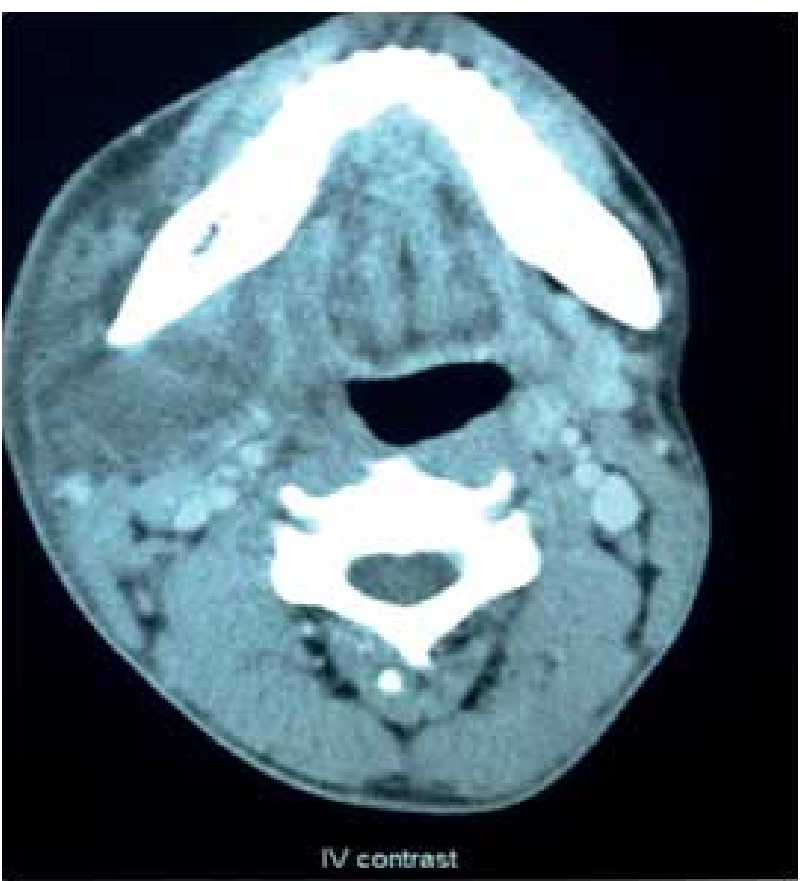

CT scan of patient case 3

Flexible nasopharyngolaryngoscopy was done and did not show any medialization. Aspiration was done intra-orally under local anesthesia, and there was minimal pus and blood stained fluid drained. Computed Tomography scan was done, revealing an abscess at angle of right mandible extending to right masticator space. Aspiration under local anesthesia was re-attempted due to persistent high spiking fever, draining 4 milliliters of pus. Patient was booked for incision and drainage transorally under general anesthesia; there was a loculated abscess medial and lateral to mandible, draining $20 \mathrm{mls}$ pus. Patient was discharge well. He was reviewed 12 days later and he well with full STO done. Patient defaulted subsequent follow up.

\section{Discussion:}

The masseteric space is formed by the superficial layer of the deep cervical fascia as it surrounds the pterygoid muscles medially and the masseter muscle laterally ${ }^{3}$. This space also contains ramus and body of the mandible ${ }^{3}$.

The masseter muscle has three parts which are superficial, middle and deep portion that originates from the zygomatic arch. ${ }^{4}$ The insertion of superficial portion is to the lower third of the ramus while the middle portion is inserted and attached to the curve thin line superiorly and posteriorly over the middle third of the ramus. ${ }^{5}$ The deep portion of masseter muscle is attached to the upper third of the ramus of mandible and the lateral aspect of coronoid process ${ }^{5}$. The different loose attachments

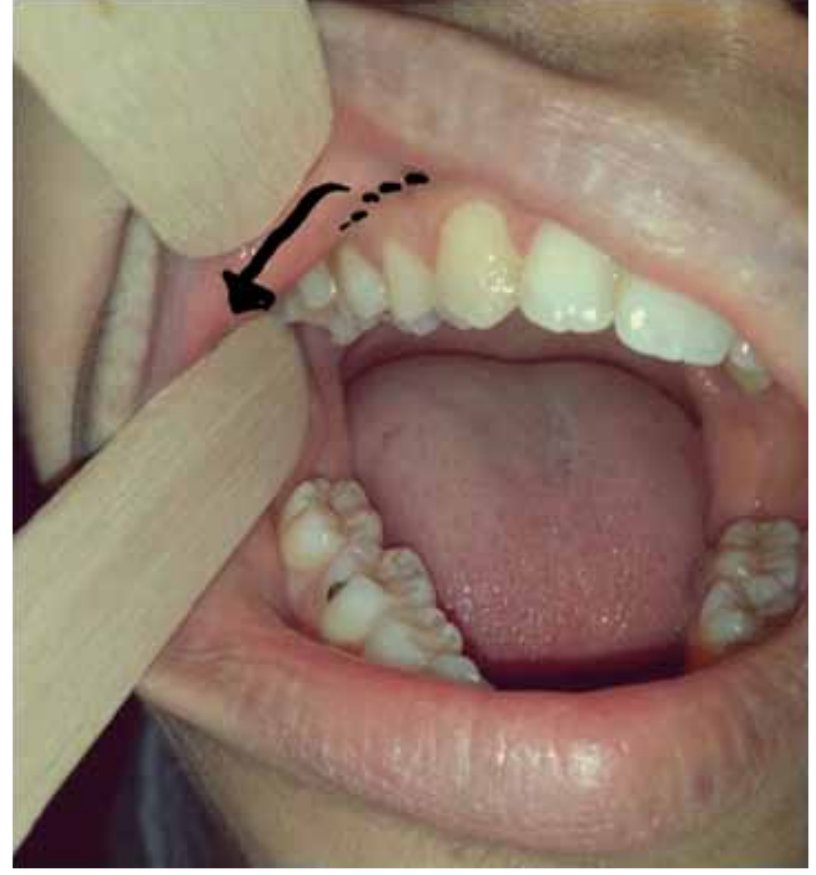

Surgical approach in our series of patients

and insertions of masseter muscle portions have created a potential space, which can allow formation of masseteric abscess ${ }^{6}$.

In trans-oral or intra-oral approach, surgical incision is made on mucosa along the anterior border of the ramus of mandible, ${ }^{7}$ and a sinus forceps is employed and inserted into the space which is lateral to the ramus of mandible and medial to masseter muscle. ${ }^{7}$ The masticator space is opened up and pus is drained out.

We approached slightly differently, by making an incision at the upper gingivo-buccal sulcus, and a plane is identified and dissected by using curved artery forceps through the sulcus into the masseter space, right up to the attachment of masseter to the ramus, and the wound left opened.

The advantages of this approach are faster wound healing and avoidance of some complications such as neuro-vascular injury ${ }^{8}$.

In our series, the wound was left open for secondary healing. We observed that by the time mucosa healing occurs which is within 3-4 days, residual pus was totally drained and inflammation too, resolved with the help of good antibiotic coverage. All the patients were discharged well and there were no recurrent abscess reported.

In conclusion, we strongly believe that this sublabial approach in the drainage of masseter abscess is more superior as it has less complications and a faster healing process. Further studies with proper statistical analysis are required to compare this 
novel approach with other intra-oral or external Conflict of interest: None approaches in draining masseter abscesses.

\section{References:}

1. Mandel L. Submasseteric Abscess Caused by a Dentigerous Cyst Mimicking A Parotitis: Report of Two Cases. J Oral Maxillofacial Surg 1997.55:996-999.

2. Gidley PW, Ghorayeb BY, Stiernberg CM. Contemporary management of deep neck space infections. Otolaryngology Head Neck Surg 1997.116:16-22.

3. Balatsouras DG, Kloutsos GM, Protopapas D, et al. Submasseteric abscess. J Laryngol Otol 2001. 115 (1): 68-70.

4. Jones KC, Silver J, Millar WS, Mandel L. Chronic Submasseteric Abscess: Anatomic, Radiologic and Pathologic features. Am J Neuroradiol 2003.24: 1159-
1163.

5. Sayuti Ra, Baharudin Aa , Amran Mb . Submasseteric abscess: an unusual head and neck condition. Archives of Orofacial Sciences 2007.2:59-60.

6. MacDougall JDB. The attachments of the masseter muscle. Br Dent J 1955. 98:193-199.

7. Srirompstong S, Srirompotong S. Surgical emphysema following intraoral drainage of buccal space abscess. $J$ Med Assoc Thai 2002 .85: 1314-1316.

8. Yadranko D, Lance O, Allison T. Transoral approach to the Superomedial Parapharyngeal Space. Otolaryngology Head and Neck Surgery 2006.134:466-470. 